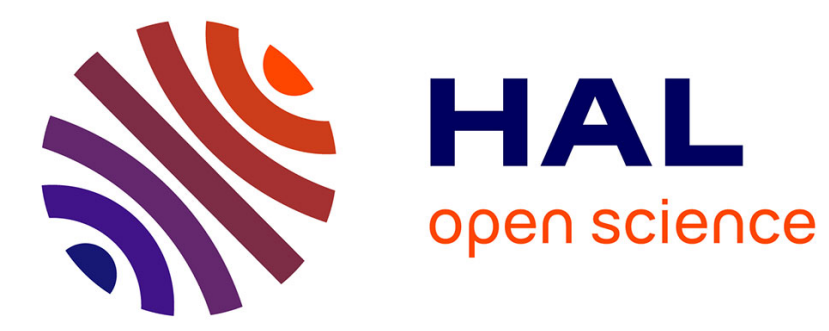

\title{
Trempe des lacunes dans le niobium
}

Claude Tete, Michel Lebouche, Bernard Deviot

\section{To cite this version:}

Claude Tete, Michel Lebouche, Bernard Deviot. Trempe des lacunes dans le niobium. Revue de Physique Appliquée, 1970, 5 (3), pp.423-425. 10.1051/rphysap:0197000503042300 . jpa-00243411

\section{HAL Id: jpa-00243411 https://hal.science/jpa-00243411}

Submitted on 1 Jan 1970

HAL is a multi-disciplinary open access archive for the deposit and dissemination of scientific research documents, whether they are published or not. The documents may come from teaching and research institutions in France or abroad, or from public or private research centers.
L'archive ouverte pluridisciplinaire HAL, est destinée au dépôt et à la diffusion de documents scientifiques de niveau recherche, publiés ou non, émanant des établissements d'enseignement et de recherche français ou étrangers, des laboratoires publics ou privés. 


\title{
TREMPE DES LACUNES DANS LE NIOBIUM
}

\author{
Claude TETE, Michel LEBOUCHE et Bernard DEVIOT \\ Laboratoire de Physique du Solide, Ecole des Mines, Nancy, E. R. A., CNRS No 102
}

\begin{abstract}
Résumé. - Deux types d'échantillons ont été étudiés : des rubans d'épaisseur 12,5 microns et des fils de 5/100 mm de diamètre. Dans les deux cas les échantillons, placés dans une ampoule en pyrex, sont dégazés sous ultra-vide à $2000^{\circ} \mathrm{C}$ puis, avant d'être scellée, l'ampoule est remplie d'hélium ultra-purifié par passage sur zéolithe à la température de l'hydrogène liquide.

Le paramètre d'étude est la résistivité ; les échantillons sont chauffés par effet Joule et trempés par coupure du courant.

Sur les rubans, les recuits isochrones mettent en évidence un seul stade ; par contre, sur les fils, on observe deux stades. Ces stades sont interprétés comme dus aux défauts ponctuels retenus par la trempe.
\end{abstract}

Abstract. - Two types of specimen have been studied : 12,5 micron thick ribbons and 5/100 mm wires. In both cases, the specimen placed in a pyrex tube were degassed in ultra-high vacuum at $2000{ }^{\circ} \mathrm{C}$ and, before sealing, the tube was filled with helium purified by passing through zeolite at hydrogen liquid temperature.

The specimens were heated by the Joule effect and quenched by cutting off the current and resistivity measurement were made.

The ribbons show only one stage of isochrone annealing while two stages were observed for the wires. These stages are considered due to point defects remaining after the quench.

Le manque de données sur les défauts ponctuels dans les métaux cubiques centrés a suscité, ces dernières années, un grand nombre de recherches sur les métaux réfractaires des groupes V A et VI A : W, Mo, $\mathrm{Ta}, \mathrm{Nb}$. C'est ainsi que des expériences de trempe ont été faites mais les seuls résultats publiés concernent le tungstène et le molybdène [1] [2]. La trempe de ces quatre métaux présente en effet quelques difficultés. Une grande vitesse de trempe est imposée par la valeur relativement faible attendue pour l'énergie de migration [3] [4]. Mais pour $\mathrm{Nb}$ (et Ta), la difficulté la plus importante vient de la grande réactivité de ce métal vis-à-vis des gaz comme l'oxygène, l'azote, l'hydrogène [5], ce qui pose le problème de la pollution par le milieu de trempe et de recuit. C'est ainsi que l'influence prépondérante de ces impuretés sur le stade III de $\mathrm{Nb}$ a été mise en évidence par deux groupes d'auteurs [6] [7]. Notre premier souci a été de distinguer l'effet des défauts ponctuels d'un effet d'impuretés.

A. Dispositif. - Parmi les méthodes de trempe en usage, la plus adéquate nous a paru être la méthode de trempe en capsule déjà utilisée par Doyama et Koehler [8] pour l'or. Nous disposons pour cela d'une installation permettant à la foi le dégazage du niobium sous ultra-vide et le remplissage de la capsule en hélium purifié.

a. Réalisation DE LA CAPSUle. - Deux rubans d'environ $6 \mathrm{~cm}$ de longueur, $0,75 \mathrm{~mm}$ de largeur et $12,5 \mu \mathrm{m}$ d'épaisseur, de niobium MRC, grade Marz, sont soudés par points sur des passages en tungstène traversant un pied en pyrex. Ce pied est ensuite soudé au tour à verre à une capsule également en pyrex. Pendant cette opération, un disque de tantale et un balayage d'argon protègent les échantillons d'une contamination excessive.

$b$. Dégazage et remplissage de la Capsule. La capsule ainsi préparée est montée sur une installation entièrement en pyrex, étuvable à $400^{\circ} \mathrm{C}$, où l'ultra-vide est réalisé par une combinaison de pompes à sorption et de pompes à vapeur de mercure. La pression est mesurée à l'aide d'une jauge de Bayard Alpert. Les échantillons sont alors dégazés. Avant d'être scellée, sur la même installation, la capsule est remplie (sous une pression d'une atmosphère) d'hélium $\mathrm{N} 55$ de l'Air Liquide, purifié par passage sur un mélange de zéolithes 3-4-5 $\AA$ maintenu à la température de l'hydrogène liquide [9] [10]. Pendant le remplissage et le scellement, la capsule plonge dans un dewar d'azote liquide. La pureté de l'hélium obtenu n'est pas mesurable mais est jugée satisfaisante d'après les courbes de recuit du niobium. Ainsi préparée, la capsule est conservée dans un bain d'azote ou d'hydrogène liquide pour diminuer la vitesse de pollution de l'hélium par les gaz désorbés de la paroi de pyrex. Dans ces conditions, un mois après sa préparation, l'hélium a gardé une pureté suffisante.

Un dégazage à $2000^{\circ} \mathrm{C}$ pendant une demi-heure sous un vide limite de $10^{-9}$ à $5 \times 10^{-10}$ torr donne un rapport de résistivités ambiante-hydrogène de l'ordre de 250 . 
B. Traitements thermiques et mesures. - $a$. LES CONDITIONS DE TREMPE. - Les trempes et recuits sont faits la capsule plongeant dans un cryostat à hydrogène liquide. Ces traitements thermiques sont faits par effet Joule. La trempe est réalisée par coupure du courant, les vitesses initiales mesurées à l'oscillographe cathodique étant de l'ordre de $60000^{\circ} / \mathrm{s}$ à $2500^{\circ} \mathrm{C}$.

b. Mesures. - Le paramètre d'étude est la résistivité. Toutes les mesures sont faites dans un bain d'hydrogène liquide par une méthode potentiométrique utilisant un Pont Cambridge à $0,1 \mu \mathrm{V}$; l'un des deux fils sert de témoin pour la correction de température du cryostat. La précision sur la résistivité est de quelques $10^{-5} \mu \Omega \mathrm{cm}$.

C. Résultats. - $a$. RubAN. - Des rubans ont été trempés depuis $2500^{\circ} \mathrm{K}$. La figure 1 représente le recuit isochrone de l'un d'eux, les durées de recuit étant de $5 \mathrm{mn}$ et le pas de $25^{\circ} \mathrm{K}$. Il apparaît un stade unique, de température caractéristique $770{ }^{\circ} \mathrm{K}$, la résistivité recuite étant $\Delta \rho_{0}=4,17 \times 10^{-3} \mu \Omega \mathrm{cm}$.

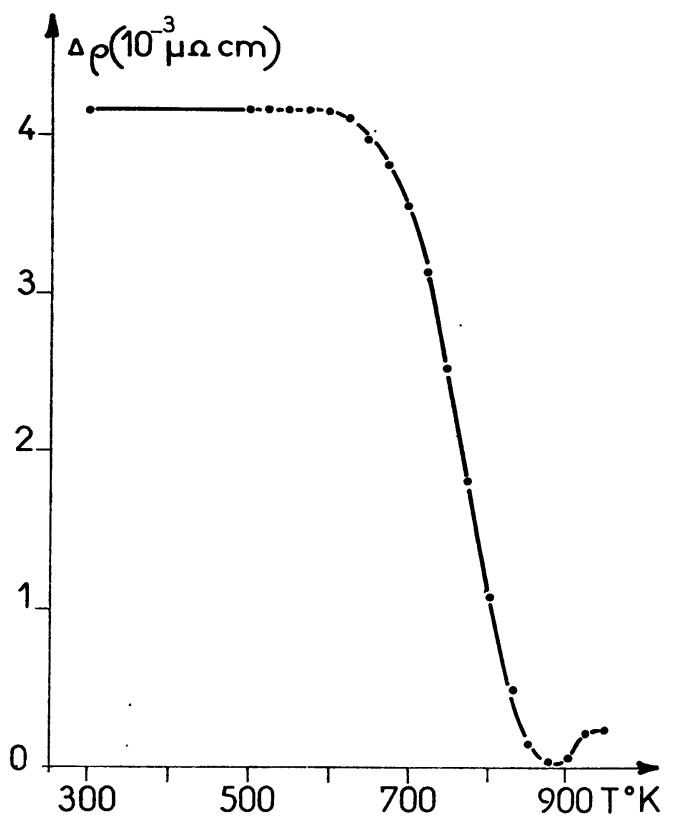

FIG. 1. - Recuit isochrone d'un ruban de niobium trempé. Température de trempe : $2500^{\circ} \mathrm{K}$. Recuits de 5 minutes tous les $25^{\circ} \mathrm{K}$

Il faut toutefois remarquer que la fin du recuit est perturbée, sans doute par une légère pollution (température supérieure à $875^{\circ} \mathrm{K}$ ). Pour le même échantillon, dans les mêmes conditions de trempe, deux recuits isothermes ont été faits, l'un à $750^{\circ} \mathrm{K}$, l'autre à $825^{\circ} \mathrm{K}$. Ils sont représentés sur la figure 2. Leur étude cinétique nous a conduits à envisager une réaction d'ordre 1 (Fig. 3) pour la plus grande partie du stade. L'énergie d'activation du stade a été déterminée par trois méthodes : à partir de l'isochrone seule dans l'hypothèse d'une réaction du premier ordre

$$
(E=0,93 \mathrm{eV})
$$

- à partir des pentes des isothermes représentées en coordonnées semi-logarithmiques $(E=0,93 \mathrm{eV})-$ par la méthode du cross-cut [11] $(0,96 \mathrm{eV}<E<1 \mathrm{eV})$.

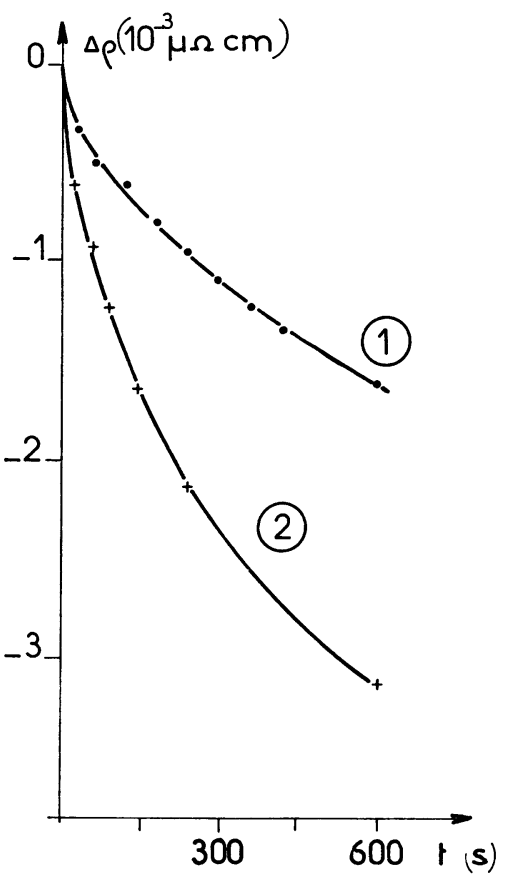

Fig. 2. - Recuits isothermes du ruban. Courbe 1 : isotherme à $750^{\circ} \mathrm{K}$. Courbe 2 : isotherme à $825^{\circ} \mathrm{K}$.

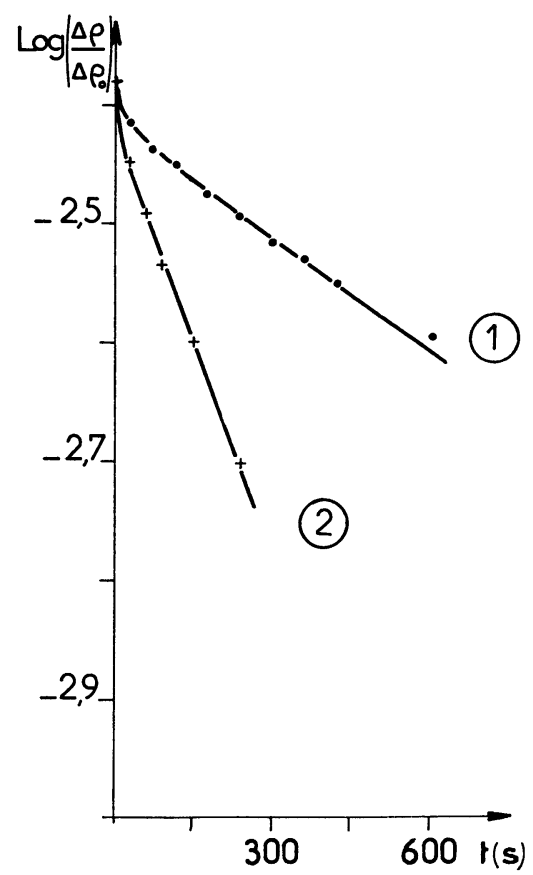

Fig. 3. - Etude des isothermes de la figure 2. Courbe 1 : isotherme à $750^{\circ} \mathrm{K}$. Courbe $2:$ isotherme à $825^{\circ} \mathrm{K}$.

b. FILs. - Un travail identique a été tenté sur des fils ; leur tendance à la rupture, due à une structure bambou, est telle que nous n'avons pas encore $\mathrm{pu}$ procéder à une étude complète. Nous pensons toutefois avoir mis en évidence deux stades de recuit, 


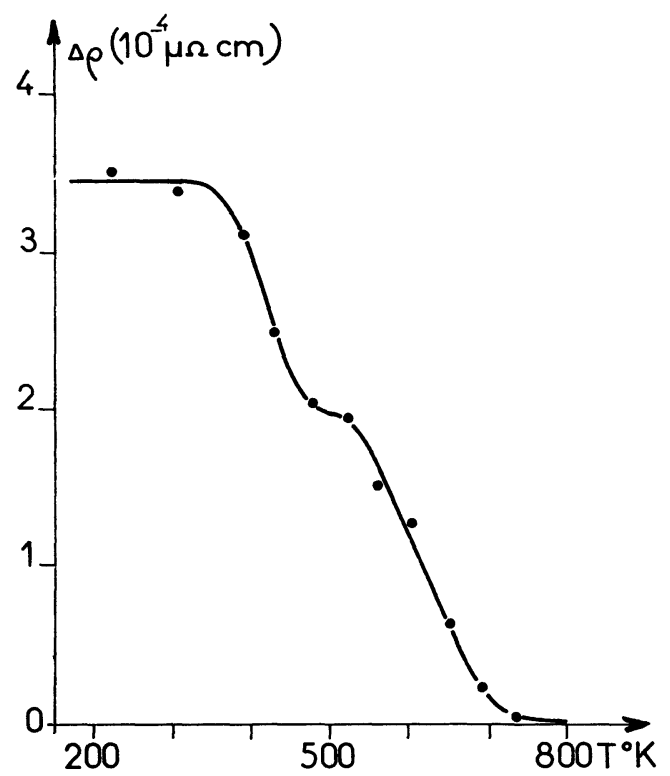

FIG. 4. - Recuit isochrone d'un fil de niobium trempé. Température de trempe : $2083^{\circ} \mathrm{K}$. Recuits de 10 minutes tous les $25^{\circ} \mathrm{K}$. visibles sur la figure 4. Celle-ci représente un recuit isochrone, avec un taux de chauffage de $10 \mathrm{mn}$ de 50 à $50 \mathrm{~K}$, pour une trempe depuis $2083 \mathrm{~K}$.

D. Discussion. - Nous pensons que la variation de résistivité observée au cours des trempes et recuits est due à des lacunes s'éliminant à la surface. En effet :

- nous avons travaillé dans des conditions de propreté meilleures que celles préconisées par Dinter pour être à l'abri des effets de pollution ;

- la cinétique de recuit est simple et du premier ordre. La distance que doit parcourir un défaut pour s'annihiler, calculée dans le cas du ruban, est d'environ $0,1 \mathrm{~mm}$, ordre de grandeur compatible avec les dimensions de l'échantillon;

- la quantité totale trempée croît avec la température de trempe;

- la valeur de l'énergie d'activation trouvée, $0,93 \mathrm{eV}$, est en accord avec le calcul théorique de Flynn pour l'énergie de migration des lacunes $(0,9 \mathrm{eV})$. Toutefois, cette valeur paraît faible, comparée aux valeurs d'énergie de formation mesurées [12] [13], pour expliquer l'énergie d'auto-diffusion [14].

\section{Bibliographie}

[1] Schultz (H.), Acta Met., 1964, 12.

[2] Gripshover, Zetto, Bass, Int. Conf. on vacancies and interstitials in metals, Julich, 1968.

[3] Gregory, Acta Met., 1963, 11.

[4] Flynn (C. P.), Phys. Rev., 1968, 171.

[5] Fromm, Jehn, Vacuum, 19.

[6] Kothe, Schlat, Phys. Stat. Sol., 1967, 21, K 73.

[7] Dinter, Z. Metallkunde, 1967, 58.

[8] Doyama, Koehler, Phys. Rev., 1962, 127.
[9] Rozenberg, KAHAN, Rapport CEA, R 3366, 1967.

[10] Hengevoss, Trans. of the third intern. vacuum Congress.

[11] Damask, Dienes, Point defects in metals, Gordon et Breach.

[12] Kraftmaker, Sov. Phys. Solid State, 1962, 5.

[13] Chekhovskol, Zhukova, Sov. Phys. Solid State, $1966,8$.

[14] Pelleg, Luidberg, Trans. AiMe, 1969, 1654. 